\title{
Transgressionen des Heiligen. Sexualität und Geschlechtlichkeit im transreligiösen Rhizom der europäischen Mystik des Mittelalters
}

\begin{abstract}
In Eros and Kabbalah, Moshe Idel speaks about „theoeroticism“ as a theological paradigm of Jewish mysticism. This concept applies to many different trends within European mysticism, as the paper shows with regard to prominent examples from the medieval mystical traditions of Judaism, Islam and Christianity. Referring to Deleuze's and Guattari's concept of the rhizome, the paper develops the idea that medieval Europe, in its political and religious heterogeneity, represented a space of rhizomorphic connections that had a larger influence on the development of mystical writing models than the traditionally invoked universality of medieval culture.
\end{abstract}

Keywords: Mittelalter; Rhizom; Religion; Erotik; Transgression; Judentum; Christentum; Islam

\section{Zur Einführung}

Der vorliegende Text wird sich mit einem Phänomen auseinandersetzen, das bei der Auslegung mystischer Texte immer wieder begegnet: der Präsenz von erotisch geprägten Ausdrucksweisen in literarischen Darstellungen der mystischen Ekstasen und Weltmodelle. Gleichzeitig will er einige Schritte weiter gehen, indem er nicht Erotik und körperliche Sinnlichkeit in der Art, wie sie im alttestamentlichen Schir ha-Schirim dargestellt wird, in den Vordergrund stellt (es sei denn in dessen radikalisierter Rezeptionsweise), sondern explizite Sexualität, solche mystischen Fantasien also, in denen Geschlechtsorgane und Sexualhandlungen literarisch inszeniert werden. In dieser Hinsicht lehnt sich der Beitrag an das intellektuelle Erbe Georges Batailles, der mit Texten wie Der heilige Eros [L'Érotisme, 1957] zu der Erörterung der hier präsentierten Thematik wesentlich beigetragen hat. Ihm wird auch der titelgebende Begriff der Transgression entnommen.

Ein weiterer - und von Bataille nicht unternommener - Schritt ist der Verzicht auf die christianozentrische Perspektive, die für die Betrachtung der religiösen Diskurse des Mittelalters in der abendländischen Tradition immer noch präsent 
ist. Und zur europäischen Mystik des Mittelalters gehört doch deutlich mehr als nur die Überlieferung aus dem Herrschaftsbereich des lateinischen Christentums. Die komparatistische Herangehensweise an christliche, islamische und jüdische Texte soll erhebliche Übereinstimmungen innerhalb dieser transreligiösen Tradition des mystischen Schreibens in Europa sichtbar machen.

Bei der Erschließung dieses interkulturellen Verknüpfungsraumes wird auf das Modell des Rhizoms verwiesen, mit dem Gilles Deleuze und Félix Guattari in den 70er Jahren des vergangenen Jahrhunderts für eine dezentrierte und enthierarchisierte Darstellung der Organisation von Wissens- und Kulturtransfer plädierten. Das mittelalterliche Europa wird somit als ein Rhizom betrachtet, in dessen Heterogenität Juden, Christen und Muslime nicht nur ausgrenzende theologische Systeme aufbauten und diese teilweise auch territorialisierten, sondern auch solche Denkmuster schufen, die im netzartigen „unterirdischen“ Geflecht von Transferwegen sich immer wieder begegneten.

\section{Phallisch-organischer "Theoerotismus“ und das göttliche Geschlecht}

Du hast also scharfsinnig zu sein, damit du die Reichweite der Liebe und den Wert der Person, die dich liebt, erkennst und auch damit du eilst, dich mit ihr zu vereinen, indem du dich über Seine Liebe mit Eigenschaften Gottes [...] tränkst. So legt Er es dir nahe. Ist Er es doch, Der dich ursprünglich mit dieser Liebe, der nie etwas gleichkommen wird, erschaffen hat. Deshalb ist der Liebesakt, wenn er auch im Grunde genommen mit ihm [jedes Mal neu] vollzogen wird, lediglich die Folge jener Liebe, mit der Er dich schon seit Anbeginn liebt. (Ibn 'Arabī 2009, 132)

In seinem Werk Al-Futūḥāt al-Makkīya [Die mekkanischen Offenbarungen] empfiehlt dies Ibn 'Arabī (1165-1240), der aus dem spanischen Murcia stammende Asch-Schaich al-Akbar (,der größte Meister“) des Sufismus, einem Menschen, der ein „brennendes Verlangen“ nach sexueller Erfüllung empfindet. Jeder Sexualakt ist demnach eine intime Begegnung mit Gott und Vergegenwärtigung der Schöpfung, welcher der erste „Coitus“ folgte (vgl. Ibn 'Arabī 2009, 132). Die Sexualisierung der Denkweise an das Verhältnis vom Gott und Mensch ist geradezu paradigmatisch für die mystischen Texte verschiedener religiöser Provenienz. Das höchste Arkanum der Mystik, die unio mystica, wird von mittelalterlichen Mystikerinnen und Mystikern gern in erotischen, ja sexuellen Bildern imaginiert, wobei ihr Erlebnis durchaus körperlich erfahren wird.

In seinen Tagebüchern stellt Bonaventura (1217-1274), der große Doctor Seraphicus der katholischen Kirche, beispielsweise fest, dass es nicht selten geschehe, 
dass diejenigen, die den mystischen Weg beschreiten, „in geistigen Leidenschaften von der Flüßigkeit des fleischlichen Ausflusses beschmutzt werden“ (Bataille 1974, 241). ${ }^{1}$ Beide Denker, der islamische Sufi-Meister wie auch der große Gelehrte der mittelalterlichen christlichen Scholastik und hohe katholische Würdenträger, lebten in der Blütezeit der mittelalterlichen Kultur, die die Zeit von der zweiten Hälfte des elften bis zum Ende des dreizehnten Jahrhunderts umschließt. Ein wenig später entstand in Spanien auch das Grundwerk der jüdischen Kabbala, Sefer ha-Sohar, das von Mosche de Leon (um 1250-1305) niedergeschriebene Buch des Glanzes, in dem sexuelle Bilder so stark präsent sind, dass der größte Kabbalaforscher der Gegenwart, Moshe Idel, in seinem einschlägigen Werk zu Eros and Kabbalah vom „theoeroticism“ als einem theologischen Paradigma der jüdischen Mystik spricht (Idel 2005, 42). Es ist ein Begriff, der allerdings in vielen unterschiedlichen Strömungen innerhalb der europäischen Mystik seine Geltung haben kann.

Im Zusammenhang damit muss über den Geschlechtscharakters des göttlichen Prinzips in mystischen Denkmodellen reflektiert werden. Idel weist darauf hin, dass den fundamentalen Unterschied zwischen der rational-philosophischen Strömung des Judaismus und dessen mystischen Formen der Glaube daran bildet, dass Gottheit dynamisch ist und beide Dimensionen - die männliche und die weibliche - in sich vereinigt, was die Harmonie des Kosmos aufrechterhält (vgl. Idel 2005, 5-56). ${ }^{2}$ In der christlichen Tradition ist das Denken der göttlichen Weiblichkeit für das persönliche Gott-Erlebnis bei Heinrich Seuse charakteristisch. Auch wenn der Gegenstand der Liebe in der mystischen Religiosität des christlichen Westens im Allgemeinen als in Christus vertretenes männliches Prinzip gedacht wird, dem die Seele in hingebender Weiblichkeit sich naht, von dem sie ergriffen und umschlungen sein möchte, neigt die weiche Seele Seuses dazu, das Göttliche weiblich zu denken (vgl. Mehlis 1927, 131). Seuse sieht in Christus oft das Ewig-Weibliche, das ihn hinanziehen soll - in mystischer Liebe (vgl. Schwarz 1934, 39-41).

1 Diese Tendenz zur Darstellung von religiösen Erlebnissen in körperlichen Bildern prägt die mittelalterlichen mystischen Vorstellungen im gravierenden Maße, wofür der Wunsch der hl. Lutgard, ihr Herz mit Christi Herz auszutauschen (wobei es nicht symbolisch, sondern durchaus organisch gemeint ist), ein weiteres Beispiel sein kann (vgl. Grzybkowski 1997, 142).

2 Auf Gershom Scholems Schechina-Essay wird im Weiteren eingegangen. 


\section{Zwischen Sacrum und Profanum}

Die Blütezeit der mittelalterlichen Mystik ist gleichzeitig dieselbe Periode, in der in Europa auch die weltliche Liebeslyrik ihren Höhepunkt erlebte, eine Lyrik, die keineswegs nur die platonische hohe Minne besang, sondern auch das erotische Verlangen und seine sexuelle Erfüllung darstellte. Dabei lässt sich eine ganze Palette der Liebesbilder zwischen der subtilen Erotik und der Obszönität nennen: Unter der linden Walthers von der Vogelweide, die Tagelieder Wolframs von Eschenbach, die Herbst- und Winterlieder Steinmars von Klingenau, das Erntelied von Johannes Handloub, wo die Einzelheiten eines Liebestreffens beschrieben sind etc. (vgl. Szyrocki 1986, 143).

Es gilt nun, die Verflechtung dieser Tendenzen in der europäischen Kultur des Hochmittelalters zu erfassen und nach möglichen Gründen der Tatsache zu fragen, dass vielerorts die gleichen Bilder sowohl im profanen Bereich als auch in den Imaginationen des absoluten Sacrums versprachlicht werden.

Wie eingangs bereits angedeutet, soll dies mit vollem Bewusstsein dessen geschehen, dass die Kultur des europäischen Mittelalters nicht eine homogene Einheit darstellte, wie es im Zuge der nationalen Mythosbildung des späten achtzehnten und des neunzehnten Jahrhunderts gesehen wurde, als Novalis etwa in seinem Fragment Die Christenheit oder Europa „die schönen, glänzenden Zeiten“ gepriesen hat, „wo Europa ein christliches Land war, wo Eine Christenheit diesen menschlich gestalteten Weltteil bewohnte“ (Novalis 2013, 499). Hält man an diesem traditionellen Klischee einer homogenen europäischen Identität der medii aevi fest, muss man weite Teile des Kontinents ausklammern, die nicht unter der Vorherrschaft des katholischen Kirchenprimats in Rom standen, darunter auch das Gebiet von Al Andalus, dem maurischen Spanien, das vom frühen achten Jahrhundert bis zu dem endgültigen Sieg der Reconquista 1492 von islamischen Machthabern regiert wurde und in dieser Zeit eine Hochkultur hervorbrachte, die weit über die Grenzen der Iberischen Halbinsel ausstrahlte. Derselbe Novalis war sich übrigens der Bedeutung der orientalen Quellen der Poesie durchaus bewusst, wie die Zulima-Episode in seinem Ofterdingen klar zu beweisen scheint (vgl. Novalis 2013, 167-177), auch wenn Zulima nicht nur als die kulturell Andere, sondern auch als weibliches Sprachrohr für männliche auktoriale Phantasien betrachten werden muss (vgl. Hodkinson 2009, 119).

Es soll hier aber nicht nach dem althergebrachten Prinzip des ex oriente lux gedacht werden, sondern es wird zu zeigen sein, dass die europäische Kultur im Mittelalter, trotz der universalistischen Ansprüche der weltlichen und religiösen Mächte, eine heterogene und rhizomorphe Struktur aufwies, in der das islamische und jüdische Gedankengut genauso ihren Platz haben wie das Weltbild des Christentums, das wiederum mindestens seit dem großen Schisma von 1054 in 
sich keineswegs einheitlich war. Der Universalismus der mittelalterlichen Kultur soll demnach nicht in der Vorherrschaft eines dominierenden Zentrums gesehen werden, sondern in der Parallelität der Erscheinungen, die sich aus einem manchmal schwer nachvollziehbaren Kulturtransfer ergab und Imaginationsstrukturen reproduzierte, die - synchron und diachron, vertikal und horizontal zerstreut an verschiedenen Orten und in unterschiedlichen Kontexten zu gleicher Zeit eine erstaunliche Relevanz gewannen. Dies entspricht vor allem den Prinzipien von Konnexion und Heterogenität, die Deleuze und Guattari für ihr Rhizom-Konzept geltend gemacht haben:

Jeder beliebige Punkt eines Rhizoms kann (und muß) mit jedem anderen verbunden werden. [...] Ein Rhizom [...] verbindet unaufhörlich semiotische Kettenglieder, Machtorganisationen, Ereignisse aus Kunst, Wissenschaft und gesellschaftlichen Kämpfen. Ein semiotisches Kettenglied gleicht einer Wurzelknolle, in der ganz unterschiedliche sprachliche, aber auch perzeptive, mimische, gestische und kognitive Akte zusammengeschlossen werden: es gibt weder eine Sprache an sich noch eine Universalität der Sprache, sondern einen Wettstreit von Dialekten, Mundarten, Jargons und Fachsprachen. (Deleuze und Guattari 1992, 16-17)

So liegt auch das Charakteristikum der hier behandelten Phänomene in der Überschneidung des Heterogenen: des Jüdischen, Islamischen und Christlichen, des sprachlichen Idioms der Mystik in seinen dichotomen Formen von ekstatischer Rede bis hin zu kühner theologischer Spekulation und der der Welt zugewandten Liebeslyrik mit ihren inhaltlichen und stilistischen Schattierungen, des Sakralen und des Profanen, des Absoluten der reinen Geistlichkeit und der Direktheit von sexuellen Phantasien.

\section{Mystische Transgressionen}

Die sexuell aufzufassende Erotik als Topos des mystischen Schreibens mag im Zusammenhang der rhizomorphen Struktur der mittelalterlichen Kultur besonders aussagekräftig erscheinen, da die Sexualität im öffentlichen, vor allem religiösen Diskurs der Zeit weitgehend tabuisiert blieb und in die Sphäre der Scham verdrängt wurde. Ihre Präsenz in der profanen Dichtung und - in einem noch höheren Maße - in religiösen Texten weist somit Symptome einer Transgressionsbewegung auf, wie sie etwa Bataille beschrieb, der glaubte, dass die Erotik für den Menschen einen Sinn hat, der mit wissenschaftlichen Methoden nicht erkundet werden kann: 
Wir sprechen immer dann von Erotik, wenn ein Mensch sich auf eine Weise verhält, die zu den gewöhnlichen Sitten und Meinungen in betontem Gegensatz steht. Die Erotik zeigt die Kehrseite einer Fassade, deren einwandfreies Äußeres nie in Abrede gestellt wird: Auf der Kehrseite enthüllen sich Gefühle, Körperteile und Gewohnheiten, deren wir uns gewöhnlich schämen. (Bataille 1974, 118)

Die erotische Erfahrung ist somit die „Erschütterung einer Ordnung“ und eine Überschreitung des Verbots einer sündhaften Lust:

Im Augenblick der Überschreitung empfinden wir die Angst, ohne die es das Verbot nicht gäbe: Das ist die Erfahrung der Sünde. Die Erfahrung führt zur vollendeten Überschreitung, zur geglückten Überschreitung, die das Verbot aufrechterhält, und zwar es aufrechterhält, um es zu genießen. Die innere Erfahrung der Erotik verlangt von dem, der sie macht, eine nicht weniger große Sensibilität für die Angst, die das Verbot begründet, wie für das Verlangen, das zu seiner Übertretung führt. (Bataille 1974, 38-39)

Auch wenn die Mystikerinnen und Mystiker ihre kühnen erotischen Bilder geistig sublimierend kommentieren, wie es etwa der bereits zitierte Ibn 'Arabì in seinen Ausführungen zu den 61 mystischen Liebesgedichten aus der Sammlung Turjumān al-Ashwāq [Deuter der Sehnsüchte] tut (vgl. Ibn 'Arabī 2013) und wie es auch bei anderen mystischen Dichtern, etwa Hildegard von Bingen (vgl. Hildegard von Bingen 1963) oder Juan de la Cruz (vgl. Bossong 2007, 104; Boldt 2013), der Fall ist, und wenn man auch nicht die mystische Ekstase eindeutig auf die unbewusst geschehende orgasmische Befriedigung herabsetzen kann, was Bataille der psychoanalytischen Schule vorwirft, etwa Marie Bonaparte, die die Transverberation der Teresa von Ávila als heftigen sexuellen Orgasmus auffasste (vgl. Bataille 1974, 224-225), kann die augenscheinliche Parallelität des mystischen Erlebnisses und der körperlichen Wollust nicht geleugnet werden. In der besagten Transverberationsszene aus Teresas Vida ist diese Parallelität von geistiger Vereinigung und körperlicher Durchdringung mit masochistischen Zügen derart evident, dass man diese Evidenz nicht übersehen kann:

Ich sah ihn mit einer langen Lanze aus Gold, und ihre Spitze war wie aus Feuer, mir schien es, als stieße er sie wiederholt in mein Herz und durchdringe es bis in meine Eingeweide! Als er die Lanze herauszog, war mir, als zöge er auch diese heraus und ließe mich ganz im Feuer der großen Gottes-Liebe. Der Schmerz war so groß, daß ich stöhnen mußte, und dennoch war die Süße dieses übermäßigen Schmerzes derart, daß ich nicht wünschen konnte, davon befreit zu sein [...]. (zit. nach: Bataille 1974, 224)

Es ist zugleich eine Beschreibung, die in ihrer Kühnheit kaum Entsprechungen in der profanen Literatur des voraufklärerischen Zeitalters hat. Die Letztere ist hauptsächlich der Versprachlichung von Wunsch und Verlangen verpflichtet, die 
religiöse Sprache der Erotik vergegenwärtigt aber deren unmittelbare Erfüllung. Der heilige Eros scheint aus den Tiefen des kollektiven Gedächtnisses der Menschheit zu schöpfen, in dem uralte Vorstellungen eines Hieros gamos und die damit verbundenen Schöpfungsmythen gespeichert bleiben. Die heilige Erotik oder um noch einmal die Formel Moshe Idels heranzuziehen - der Theoerotismus der mystischen Texte ist demnach in einem mehrfach verflochtenen Rhizom anzusiedeln. Dieses besteht in erster Linie aus transkulturellen Archetypen und deren literarischen Repräsentationen auf der diachronen Überlieferungslinie, sowie der Grunderfahrung der Sexualität des Körpers und deren geistiger Sublimierung auf der Ebene der Synchronie. Desweiteren wirken in ihm direkte und nachvollziehbare Transferprozesse zwischen unterschiedlichen Kulturen auf horizontaler Linie und nur bedingt ahnbare Reproduktionsmuster auf der vertikalen, die auf allgemein anthropologische Basistendenzen der Einbildungskraft und Welterfahrung zurückgeführt werden müssen.

\section{Radikale Lesarten des Hohenliedes und Weiblichkeitsbilder der Mystik}

Bei der vertikalen Verfolgung der rhizomatischen Verbindungslinien handelt es sich vor allem um die Relevanz des jüdischen Schir ha-Schirim, des Hohenliedes des hebräischen Tanach, das gleichermaßen intensiv sowohl von Ibn 'Arabī (vgl. Boldt 2013, 97), als auch von dem Autor des Sohar und von den christlichen Mystikern und Mystikerinnen rezipiert wurde. Ulrich Müller bemerkt,

daß die im Neoplatonismus angelegte Erotisierung der Religion gerade im 12. Jahrhundert in den Hohe-Lied-Predigten Bernhards von Clairvaux einen ersten literarischen Niederschlag fand, also gleichzeitig mit der Vergöttlichung der Frau in der weltlichen Troubadourlyrik. (zit. nach Augner 2001, 166-167)

Diese Sakralisierung des Profanen und die gleichzeitige Profanierung des Sakralen sieht Müller also in der durch die neoplatonische Tradition eingeleiteten und durch die literarischen Tendenzen der Zeit unterstützten Lektüre des Hohenliedes. Dieses Prachtstück der althebräischen Liebesdichtung, das erst recht spät, in der rabbinischen Zeit Eingang in den Kanon der jüdischen heiligen Schriften fand, bildet seinerseits ein Palimpsest, in dessen Schichten womöglich sumerische und akkadische Imaginationen der heiligen Hochzeit, altägyptische und syrische Liebeslieder und zeremonielle jüdische Hochzeitsriten mitklingen (vgl. Kramer 1962, 28-29, Kramer 1963, 489, Weinfeld 1996, 525, Schmidt 1995, 314-316). 
Schösslinge dieses verflochtenen Überlieferungsrhizoms sind sowohl in der spezifischen Weiblichkeitskonzeption des Ibn 'Arabī, als auch in den SchechinaDarstellungen im Sochar und der christlichen Brautmystik einer Mechthild von Magdeburg zu finden. Hiermit wird auf die Geschlechtlichkeit des Göttlichen zurückgegriffen.

Ibn 'Arabīs Weltverständnis gründet auf dem Konzept des Nikāh. Das Wort meint sowohl die Eheschließung als auch den Geschlechtsakt, den er nicht nur im biologischen Sinne versteht, sondern er umschließt für ihn alles Seiende als eine Vereinigung des männlichen und des weiblichen Prinzips. Alles, was ist, ist ein Resultat des Nikāh. Ibn 'Arabī unterscheidet zwischen einem natürlichen, einem geistigen und einem göttlichen Nikāh:

\footnotetext{
Man könnte mit ihm [dem Nikāh] sowohl die Fortpflanzung als auch den reinen Genuss bezwecken. Der göttliche [Nikāh] ist die Hinwendung des Wahren mit dem liebevollen Willen (al-irāda al-ḥubiyya) zu dem Kontingenten in der Sphäre der Kontingenz. Wenn der Wahre sich ihm [dem Kontingenten] zuwendet, wie wir erwähnt haben, lässt Er diesen Kontingenten werden. Aus dieser Vereinigung wird das Dasein des Kontingenten [als Kind] hervorgebracht. Die Entität des Kontingenten [welche im Wissen Gottes liegt] ist in diesem Fall die Gattin, die Hinwendung mit dem [liebevollen] Willen ist der Nikāh und das Ergebnis [dieser Vereinigung] ist die Entstehung des Daseins in dieser Entität. (zit. nach http://www. ibnarabi.de/pdf/Kapitel\%20198.pdf [22. April 2018])
}

Der islamische Theologe Ali Ghandour kommentiert es wie folgt: „In der Relation zu Gott sind für Ibn al-'Arabī alle Seienden weiblich, denn sie sind in dieser Beziehung in einer empfänglichen Position. Das ist, genauer gesagt, das genuine ontologische Geschlecht aller Seienden“ (http://www.ibnarabi.de/pdf/Kapitel\%20 198.pdf [22. April 2018]).

Ähnliche Vorstellungen begegnen auch in der kabbalistischen SchechinaKonzeption, die das weibliche Prinzip in der Gottheit darstellt. Die Schechina wird mit der zehnten Sefira des Lebensbaums identifiziert, die das Reich des Geschaffenen repräsentiert. Das Buch des Glanzes setzt sie in Verbindung mit der neunten Sefira, welche für den Gerechten (zaddik) steht und dem Phallus im menschlichen Körper entspricht. Sie bildet einen Kanal, dessen Passierbarkeit für den Erhalt des gesamten kosmischen Etz Chaim verantwortlich ist, da sie das Herunterfließen der Lebenssäfte in die zehnte Sefira ermöglicht. In der himmlischen Hochzeit des Gerechten mit der Schechina wird der heilige Influxus aus den oberen Sefirot in Form von Samen in den Schoß der Schechina geleitet, was die Zirkulation der Lebenskraft im gesamten Baumorganismus aufrechterhält (vgl. Scholem 1977, 135-191). Gäbe es nicht diese sexuelle (sexuell imaginierte) Verbindung, könnte die Welt nicht bestehen. 
Die heilige Erotik hat dagegen in der christlichen Erlebnismystik des Mittelalters keine so stark ontologische Ausprägung. Sie bezieht sich viel mehr auf die Sphäre eines intimen Erlebnisses der Gottesnähe, nimmt dabei aber auch viel radikalere Ausdrucksformen an, als es im Hohenlied oder bei Ibn 'Arabī etwa der Fall ist. Im XXV. Kapitel des 2. Buches von Mechthilds von Magdeburg Das fließende Licht der Gottheit spricht Gott zu der von der Liebessehnsucht ergriffenen Seele:

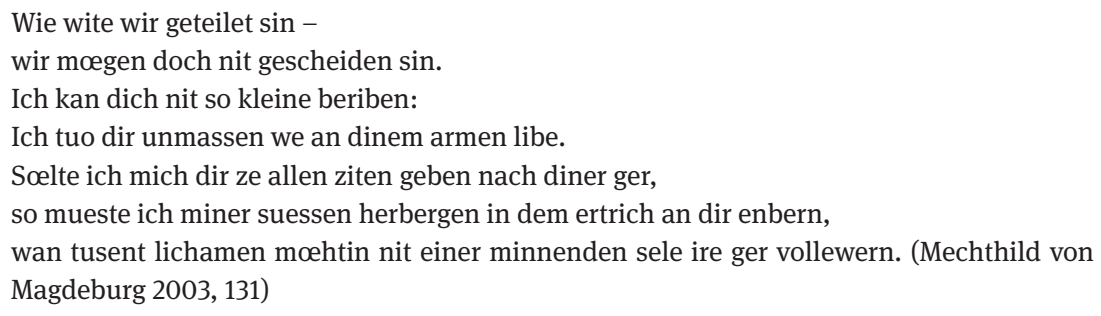

Wie Peter Dinzelbacher bemerkt, wird hier Gott selbst ,als von leidenschaftlicher Lust ergriffen imaginiert und verwendet sogar das Wort ,bereiben', ein recht konkretes Verb für den Geschlechtsverkehr“ (Dinzelbacher 2012, 100).

Hildegard von Bingen, die wohl wichtigste und einflussreichste deutsche Mystikerin des Hochmittelalters, umschreibt ihre Visionen mitunter in stark genitalen Metaphern, wie etwa in der dritten Vision des ersten Buches von Scivias:

Darauf sah ich ein riesenhaftes Gebilde, rund und schattenhaft. Wie ein Ei spitzte es sich oben zu, wurde in der Mitte breiter und nach unten zu wieder schmäler. Seine äußerste Schicht ringsum war lichtes Feuer. Darunter lagerte eine finstere Haut. [...] Unter der finsteren Haut flutete der reinste Äther. [...] Unterhalb des Äthers sah ich dunstige Luft und darunter eine weiße Haut. Der Dunst flutete hin und her und versorgte das ganze Gebilde mit Feuchtigkeit. Manchmal ballte er sich plötzlich zusammen. Dann entströmten ihm heftig rauschende Platzregen. (Hildegard von Bingen 1963, 109-110)

Auf eine textgerechte Interpretation der angeführten Darstellung sei hier aus Platzgründen verzichtet. Es sei nur - in Bezug auf die rhizomorphe Struktur der Intermedialität - auf die Tatsache verwiesen, dass die im Wiesbadener Codex enthaltene Miniatur zur Illustration dieser Vision ein geradezu anatomisches Bild der weiblichen Vagina präsentiert [s. Abb. 1].

Aloys Henning schreibt dazu:

Der Kosmos wird von der Seherin archaisch eiförmig beschrieben. Neben den Anklängen an morphologische Aspekte eines Hühnereis zeigt die Rupertsberger Kosmos-Miniatur weit mehr als andere Darstellungen die symbolische Anlehnung an die menschliche Vulva, das weibliche Geschlecht. Die goldenen Flammen der äußeren Umrandung dieses weiblichen 


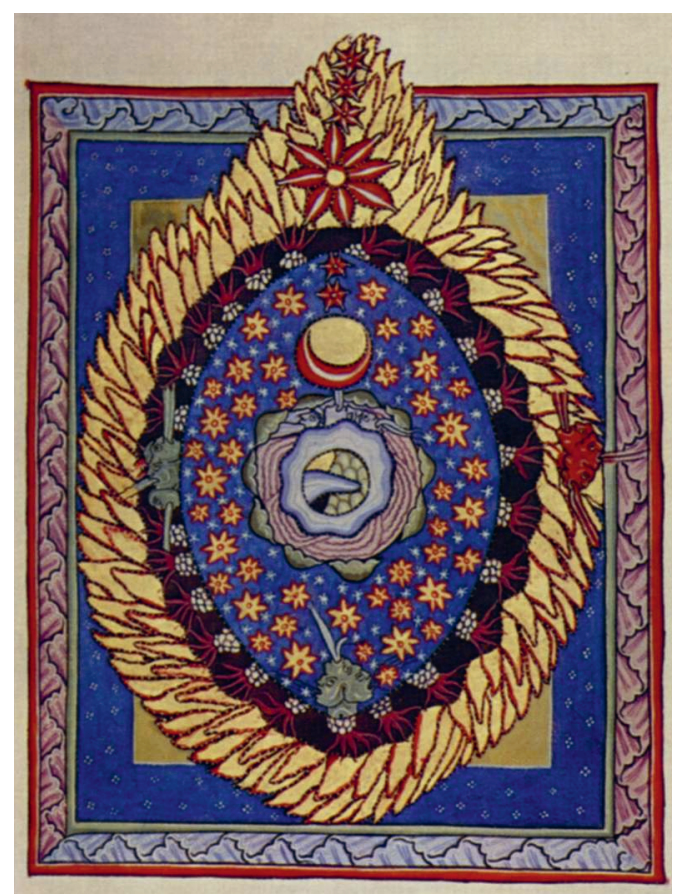

Abb. 1: Miniatur zur dritten Vision aus Hildegards von Bingen Scivias (Rupertsberger Riesenkodex). https://commons.wikimedia.org/wiki/File:Meister_des_Hildegardis-Codex_001.jpg

Symbols - die Aureole - verweisen auf seine Göttlichkeit. Die Anordnung der Sonne als ,rötlich funkelnder Feuerball, so groß, daß das ganze Gebilde von ihm sein Licht empfing‘ im oberen Drittel dieses Kosmos ist unschwer am anatomischen Ort der Clitoris zu erkennen. Die Theologie der Inkarnation Gottes [...] fußt bei Hildegards Vision auf Erfahrung weiblicher Sexualität, wie Scivias-Texte ausweisen. In ihnen benutzt die Seherin als kosmische Chiffren [...] emotionale und physiologische Aspekte weiblicher Sexualität - Anspielungen auf Orgasmen und Menstruation. (Henning 2002, 497-498)

\section{Der asignifikante (?) Bruch}

An dieser Stelle seien die Erörterungen unterbrochen. Wenn auch Deleuze und Guattari vom Prinzip eines „asignifikanten Abbruchs“ (Deleuze und Guattari 1992, 19) ausgehen, diagnostizieren sie eine signifikante Unterbrechung des Rhizoms, die immer eine unerwartete Fortsetzung verspricht: „Ein Rhizom kann an jeder Stelle unterbrochen und zerrissen werden, er setzt sich an seinen eigenen oder an anderen Linien weiter fort.“ (Deleuze und Guattari 1992, 19) In dem Sinne 
bedeutet jeder solche Bruch etwas, nämlich die Möglichkeit der Fortsetzung und Wiederaufnahme.

In der Tat - die Erforschung des hier nur skizzenhaft angeschnittenen Sachverhalts bedürfte einer Fortsetzung und einer Wiederaufnahme auf dem Niveau einer interdisziplinären Komparatistik. Ein Rhizom lässt sich wohl nur rhizomatisch erfassen.

\section{Literaturverzeichnis}

Augner, Christine. Gedichte der Ekstase in der Literatur des 16. und 17. Jahrhunderts. Tübingen: Gunter Narr, 2001.

Bataille, Georges. Der heilige Eros. Übers. v. Max Hölzer. Frankfurt/M.: Ullstein, 1974.

Boldt, Johannes. Gotttrunkene Poeten: Juan de la Cruz und die Sufi-Mystik. Münster: Lit, 2013.

Bossong, Georg. Das maurische Spanien: Geschichte und Kultur. München: 2007.

Deleuze, Gilles, und Felix Guattari. Tausend Plateaus. Kapitalismus und Schizophrenie. Übers. v. Gabriele Ricke und Ronald Voullié. Berlin: Merve, 1992.

Dinzelbacher, Peter. Deutsche und niederländische Mystik des Mittelalters. Ein Studienbuch. Berlin und Boston: De Gruyter, 2012.

Ghandour, Ali. Die Weiblichkeit bei Muhyī ad-Dīn Ibn al-Arabī. http://www.ibnarabi.de/pdf/ Das\%20Weibliche\%20in\%20den\%20theologischen\%20Lehren\%20Muhy\%C4\%AB\%20 ad-D\%C4\%ABn\%20lbn\%20al-\%CA\%BFArab\%C4\%AB.pdf (22. April 2018).

Grzybkowski, Andrzej. Między forma a znaczeniem. Studia z ikonografii architektury i rzeźby gotyckiej. Warszawa: Wydawnictwo DIG, 1997.

Henning, Aloys. „Zur weiblichen Metaphorik des Auges als Angstchiffre“. Europa in der frühen Neuzeit. Festschrift für Günter Mühlpfordt. Hg. E. Donnert. Bd. 6: Mittel-, Nord- und Osteuropa. Köln, Weimar und Wien: Böhlau, 2002. 481-528.

Hildegard von Bingen. Scivias - Wisse die Wege. Übers. v. Maura Böckeler. Salzburg: Otto Müller, 1963.

Hodkinson, James. „Moving beyond the Binary? Christian-Islamic Encounters and Gender in the Thought and Literature of German Romantism“. Encounters with Islam in German Literature and Culture. Hg. James Hodkinson und Jeffrey Morrison. Rochester - New York: Camden House, 2009. 108-127.

Ibn 'Arabī, Muhyīddīn. Abhandlung über die Liebe. Aus den Futūḥāt al-Makkīyah. Übers.v. Maurice Gloton und Wolfgang Herrmann. Zürich: Chalice, 2009.

Ibn 'Arabī, Muhyīddīn. Deuter der Sehnsüchte. Übers. v. Wolfgang Herrmann. 0. O.: Edition Shershir, 2013.

Idel, Moshe. Kabbalah and Eros. New Haven: Yale University Press, 2005.

Kramer, Samuel Noah. „Cuneiform Studies and the History of Literature: The Sumerian Sacred Marriage Texts“. Proceedings of the American Philosophical Society, Vol. 107, No. 6, Cuneiform Studies and the History of Civilization (Dec. 20, 1963). 485-527.

Kramer, Samuel Noah. „The Biblical Song of Songs and Sumerian Love Songs“. Expedition 5.1 (1962): 25-31. 
Mechthild von Magdeburg. Das fließende Licht der Gottheit, Frankfurt/M.: Deutscher Klassiker, 2003.

Mehlis, Georg. Die Mystik in der Fülle ihrer Erscheinungsformen in allen Zeiten und Kulturen. München: F. Bruckmann, 1927.

Müller, Ulrich. „Mechthild von Magdeburg und Dantes Vita Nuova, oder Erotische Religiosität und religiöse Erotik“. Liebe als Literatur. Aufsätze zur erotischen Dichtung in Deutschland. Hg. Rüdiger Krohn. München: C.H. Beck, 1983.

Novalis. Werke. München: C.H. Beck, 2013.

Schmidt, Werner H. Einführung in das Alte Testament. Berlin: De Gruyter, 1995.

Scholem, Gershom. Von der mystischen Gestalt der Gottheit. Studien zu Grundbegriffen der Kabbala. Frankfurt/M.: Suhrkamp, 1977.

Schwarz, Richard. Das Christusbild des deutschen Mystikers Heinrich Seuses. Eine Begegnung von Germanentum und Christentum. Greifswald: Bamberg, 1934.

Shaikh, Sa'diyya. Sufi Narratives of Intimacy: Ibn 'Arabī, Gender, and Sexuality. Chapel Hill: The University of North Carolina Press, 2012.

Stammler, Wolfgang. Wort und Bild. Studien zu den Wechselbeziehungen zwischen Schrifttum und Bildkunst im Mittelalter. Berlin: Erich Schmidt, 1962.

Szyrocki, Marian. Die deutschsprachige Literatur von ihren Anfängen bis zum Ausgang des 19. Jahrhunderts. Warszawa: PWN, 1986.

Weinfeld, Moshe. „Feminine Features in the Imagery of God in Israel: The Sacred Marriage and the Sacred Tree“. Vetus Testamentum 46.4 (1996): 515-529.

Weiss, Bardo. Die deutschen Mystikerinnen und ihr Gottesbild. Vol. 3: Das Gottesbild der deutschen Mystikerinnen auf dem Hintergrund der Mönchstheologie. Paderborn, München, Wien und Zürich: Ferdinand Schöningh, 2004. http://www.ibnarabi.de/pdf/ Kapitel\%20198.pdf (22. April 2018).

Joanna Godlewicz-Adamiec arbeitet am Institut für Germanistik der Universität Warschau; Autorin zahlreicher Publikationen zur Kultur des Mittelalters sowie der Wechselwirkung zwischen Literatur und bildenden Künsten; seit 2015 Leiterin der Interdisziplinären Forschungsgruppe zur Kultur des Mittelalters und der Frühen Neuzeit; seit 2015 Co-Leiterin des internationalen Forschungsprojektes „Literatur - Kontexte“.

Paweł Piszczatowski arbeitet am Institut für Germanistik der Universität Warschau. Er promovierte 2003 über Lessings theologisch-kritische Schriften. 2015 habilitierte er sich zum Problem der apophatischen Sprache in der Dichtung Paul Celans. Seine Forschungsinteressen umfassen die deutschsprachige Lyrik nach 1945, Grenzgebiete zwischen Literatur, Theologie und Philosophie sowie die Rezeption der Literatur des Mittelalters, insbesondere der mystischen Apophatik, in der Zeit nach der Aufklärung. 